\title{
L-Selectin Is Down-regulated in Umbilical Cord Blood Granulocytes and Monocytes of Newborn Infants with Acute Bacterial Infection
}

\author{
CHRISTOPH BÜHRER, JOHANNES GRAULICH, DIETGER STIBENZ, \\ JOACHIM W. DUDENHAUSEN, AND MICHAEL OBLADEN \\ Departments of Neonatology, Children's Hospital, and Obstetrics, Women's Hospital, Rudolf \\ Virchow Medical Center, Berlin Free University, D-14059 Berlin, Germany
}

\begin{abstract}
The leukocyte glycoprotein L-selectin mediates an early
step in the recruitment of leukocytes to sites of inflamma-
tion. L-Selectin surface expression is rapidly down-
regulated by inflammatory signals in vitro. In a prospective
study, we found L-selectin expression on umbilical cord
blood granulocytes and monocytes to be significantly de-
creased in newborn infants with acute bacterial infection
compared with controls $(p<0.01)$. A significantly reduced
L-selectin expression of both granulocytes and monocytes
was also found to be associated with an increased neutro-
phil immature/total ratio ( $p<0.01)$ but not with other
laboratory markers of neonatal sepsis. There was no ap-
parent impact of prematurity, low birth weight, gestational
hypertension, or gestational diabetes on L-selectin expres-
\end{abstract}
ABSTRACT

L-Selectin (CD62L) is a 90 - to $100-\mathrm{kD}$ surface glycoprotein constitutively expressed by mammalian leukocytes. L-Selectin is critically involved in initiating reversible attachment of neutrophils and monocytes to activated endothelium under nonstatic conditions ("rolling") $(1,2)$. Leukocyte rolling in inflamed venules and extravasation of neutrophils into inflammatory sites are inhibited by blocking L-selectin (3-5). In addition, L-selectin is involved in neutrophil homotypic aggregation (6) and neutrophil adherence to activated platelets (7). A differentially glycosylated form of L-selectin expressed on lymphocytes mediates lymphocyte homing to lymph nodes and gut-associated lymphoid tissue (8) as well as sites of chronic inflammation (9).

Expression of L-selectin on neutrophils and monocytes is rapidly down-regulated by inflammatory signals in $v i$ tro, possibly after a short-lived transient rise in L-selectin affinity to endothelial carbohydrate structures serving as counterreceptors (10). L-Selectin down-regulation occurs

Received January 12, 1994; accepted July 7, 1994.

Correspondence: Dr. Christoph Bührer, Universitätsklinikum Rudolf Virchow, Kinderklinik, Heubnerweg 6, D-14059 Berlin, Germany.

Supported by a grant from the German Research Council (DFG Bu 623/2-2). sion. Although the mode of delivery did not affect granulocyte $\mathrm{L}$-selectin expression, umbilical cord blood monocytes showed an increased L-selectin expression after emergency cesarean delivery compared with samples obtained after elective cesarean or vaginal delivery $(p<$ $0.01)$. We conclude that acute systemic inflammation results in down-regulation of granulocyte and monocyte L-selectin expression in vivo similar to that observed in vitro. (Pediatr Res 36: 799-804, 1994)

CRP, C-reactive protein

\section{Abbreviations}

FMLP, N-formyl-methionyl-leucyl-phenylalanine I/T, immature/total via proteolytic cleavage from the cell surface (11). Agents eliciting L-selectin down-regulation from neutrophils in vitro include the bacterial chemotactic peptide FMLP, cytokines such as granulocyte-macrophage colonystimulating factor, tumor necrosis factor- $\alpha$, IL-8, neutrophil-activating peptide- 2 , and other soluble chemoattractants, e.g. the activated complement fragment C5a and leukotriene B4 (12-14). Granulocyte colony-stimulating factor and interferon- $\gamma$ exert little influence on neutrophil L-selectin expression, and there are conflicting data on neutrophil L-selectin down-regulation elicited by IL-1, platelet-activating factor, and lipopolysaccharides (12, $13,15,16)$. Both granulocyte-macrophage colonystimulating factor and $\mathrm{C} 5 \mathrm{a}$ induce L-selectin downregulation also in monocytes $(13,17)$, whereas monocyte L-selectin expression is not affected by the monocytespecific chemotaxin monocyte chemoattractant protein-1 (18).

Baseline granulocyte L-selectin expression and FMLPinduced down-regulation have been reported to be diminished in full-term healthy newborn infants compared with adults $(19,20)$, whereas no difference in L-selectin surface reactivity was found in fetal neutrophils (21) and neonatal 
monocytes (20) compared with adult cells. No data were available on the impact of conditions associated with activation of the immune system in vivo on L-selectin expression of neonatal phagocytic leukocytes. The aim of the prospective study presented here was to determine whether L-selectin surface expression of circulating granulocytes or monocytes is down-regulated in newborns with acute bacterial infection in vivo.

\section{METHODS}

Subjects. Umbilical venous cord blood was collected from 52 girls and 75 boys born at a tertiary perinatal center, most of whom had pediatric attendance at birth and who are not representative of infants born during the study period in this hospital. Pregnancies had been complicated by multiple gestation $(n=24)$, gestational hypertension $(n=7)$, gestational diabetes $(n=10)$, and premature labor activity leading to administration of fenoterol $(n=14)$ and betamethasone $(n=33)$ to the mother. Maternal laboratory markers associated with suspected amnion infection syndrome were increased CRP serum levels ( $>30 \mathrm{mg} / \mathrm{L}, n=11)$, peripheral leukocytosis $(\geq 15000 / \mu \mathrm{L}, n=15)$, and fever (rectal temperature $\geq 37.9^{\circ} \mathrm{C}, n=13$ ) before birth; 23 mothers received i.v. antibiotic treatment with cefotiam. Forty-four infants were born prematurely (gestational age $<37 \mathrm{wk}$ ); 12 had a birth weight of $<1500 \mathrm{~g}$. Cardiotocography suggested fetal distress in 27 cases. Seventy-three infants were born vaginally (28 assisted by forceps or vacuum extraction), 44 by elective cesarean delivery, and 10 by emergency cesarean delivery. Rupture of the membranes more than $24 \mathrm{~h}$ before birth occurred in 14 newborns and asphyxia (umbilical cord artery $\mathrm{pH}<7.15$ ) in six; 37 infants were born from meconium-stained amniotic fluid.

Admission to the children's hospital was initiated in the delivery room in 53 newborns. Following institutional guidelines, blood was obtained by peripheral venipuncture for bacterial culture, white blood cell count, differential blood cell count, platelet count, and CRP serum level. Another blood culture was obtained from the freshly cut umbilical cord after local disinfection with $70 \%$ isopropanol. Antibiotic treatment was given at the discretion of the attending physician after cultures had been taken. In newborns who were clinically unstable or who received antibiotics, blood examinations were routinely repeated $18-24 \mathrm{~h}$ later and thereafter whenever appropriate.

Seventy-four newborns without clinical symptoms were placed under surveillance at the nursery of the women's hospital. In 42 of these newborns having risk factors such as maternal fever, prolonged rupture of membranes, or meconium-stained amniotic fluid, clinical surveillance was augmented by blood laboratory workup and bacterial cultures. Six of these infants were admitted to the children's hospital during the first $24 \mathrm{~h}$ after symptoms developed at the postnatal ward, with subsequent sepsis workup as described above. A total of 38 new- borns from both groups were put on i.v. antibiotics during the first $24 \mathrm{~h}$ of life.

For the purpose of this study, acute bacterial infection was diagnosed if clinical symptoms leading to the institution of antibiotics within the first $24 \mathrm{~h}$ of life were confirmed by a positive blood culture and at least one pathologic laboratory parameter of the following four (22): absolute neutrophil count $\leq 2000 / \mu \mathrm{L} ; \mathrm{I} / \mathrm{T}$ ratio $\geq$ 0.2 (first sample obtained during the first $24 \mathrm{~h}$ of life; neutrophils were classified as bands if no nuclear segmentation was present or the width of the nucleus at any constriction was more than one third the width at its widest portion) (23); CRP serum level $\geq 10 \mathrm{mg} / \mathrm{L}$ (peak during the first $36 \mathrm{~h}$ of life) (24); and platelet count < $150000 / \mu \mathrm{L}$ (nadir during the first $72 \mathrm{~h}$ of life) (25). When antibiotics had been administered i.v. to the mother of the baby for suspected amnion infection syndrome before delivery, acute bacterial infection was also assumed in culture-negative newborns undergoing subsequent antibiotic treatment if two or more of the laboratory parameters outlined were pathologic.

Of the 127 newborns studied, 38 were defined as normal, healthy, term newborns (no gestational hypertension or diabetes, vaginal delivery, umbilical artery $\mathrm{pH} \geq$ 7.15 , gestational age $37-42 \mathrm{wk}$, no admittance to the children's hospital, no antibiotic treatment of the newborn). For comparison, venous blood of 28 healthy adult volunteers was collected and analyzed alongside neonatal samples.

L-Selectin expression. Blood was prevented from clotting by addition of sodium citrate and processed within 6 $\mathrm{h}$ after delivery. Fifty $\mu \mathrm{L}$ of blood were incubated for 30 min on ice with $15 \mu \mathrm{L}$ of FITC-coupled MAb anti-Leu8 directed against human $\mathrm{L}$-selectin or an $\operatorname{IgG} 2$ isotype control (Becton Dickinson, Heidelberg, Germany). Then a commercially available, formaldehyde-diethylene glycol-based fixing and lysing solution (FACS lysing solution, Becton Dickinson) was added for $10 \mathrm{~min}$. Samples were processed as specified by the manufacturer's instructions and subjected to a final fixation with $1 \%$ paraformaldehyde. Cells were analyzed on a FACScan flow cytometer (Becton Dickinson) within $48 \mathrm{~h}$ after fixation. Lymphocytes, granulocytes, and monocytes were gated on forward and side scatter diagrams. A total of 10000 events, and a minimum of 2000 events in the monocyte gate, were recorded. The instrument was subjected to weekly calibrations to ensure comparability of the fluorescence measurements performed on a log scale with 1 024-channel resolution. L-Selectin expression was calculated in arbitrary units representing the difference between the median fluorescence of the FITC-Leu8-stained and the FITC-IgG2 control sample.

Statistical analysis. Differences between two or more groups were compared by the Mann-Whitney U-Wilcoxon rank sum W test or the Kruskal-Wallis oneway analysis of variance (for numerical variables) or by the $\chi^{2}$ test (for categorical variables), with Fisher exact values being used if one expected cell value was $\leq 5$. 
Analysis of variance was used to simultaneously assess the effects of multiple factors. $p \geq 0.05$ was regarded as not significant.

\section{RESULTS}

Technical limitations. A variably increased resistance of neonatal red blood cells to the lysing method used was found to result in considerable contamination of the lymphocyte gate with residual red blood cells, whereas adult red blood cells were lysed completely by the same procedure. Therefore, L-selectin expression of lymphocytes could not be accurately determined and was omitted.

In the granulocyte gate, no effort was made to discriminate between neutrophils and eosinophils. In WrightGiemsa-stained smears, eosinophils composed $0-24 \%$ (median 2\%) of granulocytes, and the eosinophil percentage of granulocytes was not found to be related to acute bacterial infection, $\mathrm{I} / \mathrm{T}$ ratio, tocolysis, gestational age, or granulocyte L-selectin expression.

Patients with acute bacterial infection. A total of nine newborns met the criteria set for acute bacterial infection. Group B streptococcus and Escherichia coli grew in blood cultures obtained from two newborns each of these nine. In five newborns with clinical and laboratory signs of acute bacterial infection, the causative agent could not be identified after previous administration of i.v. antibiotics to the mother within $12 \mathrm{~h}$ before delivery.

Enterococcus and Proteus vulgaris were isolated from cord blood cultures of one newborn each, neither of whom had clinical or laboratory signs of infection. Both remained stable throughout the neonatal period without administration of antibiotics; the bacteria isolated were regarded as contamination, and the babies were assigned to the control group.

The group of newborns with acute bacterial infection did not differ significantly from the control group with respect to maternal age, fetal sex, multiple gestation, preeclampsia, gestational diabetes, administration of betamethasone or fenoterol, cardiotocographical signs of fetal distress, prolonged rupture of membranes, mode of delivery, prepartal maternal temperature, maternal white blood cell count or maternal CRP serum level, presence of meconium in the amniotic fluid, umbilical artery $\mathrm{pH}$, gestational age, or birth weight. However, six of nine mothers in the group of children with acute bacterial infection had received antibiotics i.v. for suspected amnion infection syndrome, compared with 17 of 118 in the control group, and five newborns with acute bacterial infection were found to be large for date, compared with 14 in the control group $(p<0.001)$. Neonatal blood analysis showed infants with infection to have a higher $\mathrm{I} / \mathrm{T}$ ratio, a lower granulocyte L-selectin expression (both $p<0.001$ ), a lower monocyte $\mathrm{L}$-selectin expression, a lower hematocrit (both $p<0.01$ ), a higher peak CRP level, a lower absolute granulocyte count, and a lower platelet count nadir (all $p<0.05$ ).
L-Selectin expression. Umbilical cord blood granulocytes and monocytes of newborns with acute bacterial infection expressed significantly less L-selectin than controls (Figs. 1 and 2). Median granulocyte L-selectin expression (median relative fluorescence) in newborns with acute bacterial infection $(n=9)$ was 298, compared with 351 in controls (total group, $n=118$ ) and 345 in normalterm healthy newborns $(n=38, p<0.001$ for both comparisons). Median monocyte L-selectin expression in newborns with acute bacterial infection was 223 , compared with 308 in controls and 307 in normal-term healthy newborns $(p<0.01$ for both comparisons). There was no difference in granulocyte or monocyte L-selectin expression between the total control group, normal-term healthy newborns, and the control group with the later excluded $(p>0.1)$.

Newborns with a pathologic $\mathrm{I} / \mathrm{T}$ ratio $(\geq 0.2)$, regarded as the single most sensitive routine laboratory test in early-onset sepsis scores $(22,26)$, had a significantly $(p<$ 0.01 ) lower L-selectin expression of granulocytes (median fluorescence: 293 versus 350) and monocytes (median fluorescence: 223 versus 308 ). Granulocyte L-selectin expression was also found to be decreased (median fluorescence: 297 versus 350) in newborns of mothers with elevated CRP serum protein levels before delivery $(>30$ $\mathrm{mg} / \mathrm{L}, p<0.05$ both by single- and multiple-factor comparison analysis), whereas no association was found between maternal CRP serum protein level before birth and umbilical cord blood monocyte L-selectin expression.

By single- or multiple-factor comparison analysis, no difference of umbilical cord blood granulocyte or monocyte L-selectin expression was found in infants of groups characterized by one of the following data, compared with the corresponding controls: gender, prematurity (gestational age $<37 \mathrm{wk})$, very low birth weight $(<1500$ $\mathrm{g})$, gestational diabetes, gestational hypertension, multiple gestation, prolonged rupture of membranes $(>24 \mathrm{~h})$, administration of cefotiam or betamethasone to the

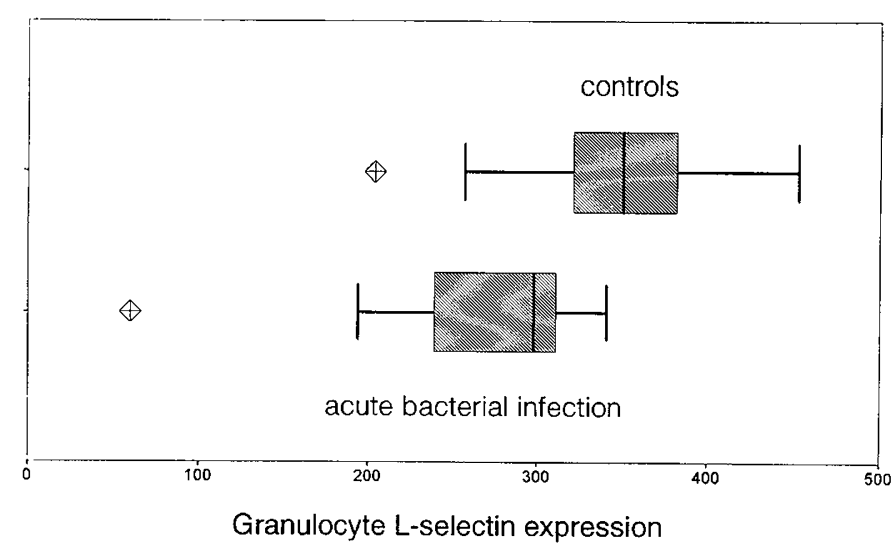

Figure 1. Granulocyte L-selectin expression (relative fluorescence intensity, arbitrary units) of newborns with acute bacterial infection (bottom, $n=9$ ) and newborns of the control group (top, $n=118$ ). Boxes contain $50 \%$ of values (25th to 75 th percentile range), with bars indicating median, whiskers showing extremes, and diamonds denoting outliers. 


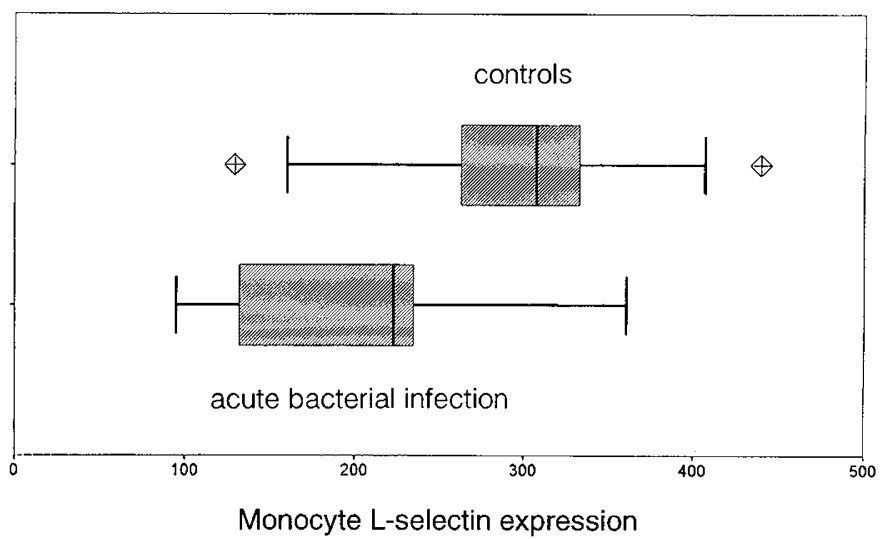

Figure 2. Monocyte L-selectin expression (relative fluorescence intensity, arbitrary units) of newborns with acute bacterial infection (bottom, $n=9$ ) and newborns of the control group (top, $n=118$ ). Boxes contain $50 \%$ of values (25th to 75 th percentile range), with bars indicating median, whiskers showing extremes, and diamonds denoting outliers.

mother before delivery, maternal fever $\left(\geq 37.9^{\circ} \mathrm{C}\right)$, cardiotocographical signs of fetal distress, asphyxia (umbilical artery $\mathrm{pH}<7.15$ ), neutropenia (absolute neutrophil count $\leq 2000 / \mu \mathrm{L}$ on first day of life), low white blood cell count $(\leq 5000 / \mu \mathrm{L})$, hematocrit $>60 \%$ or $<45 \%$, thrombocytopenia (platelet count nadir $<150000 / \mu \mathrm{L}$ within first $72 \mathrm{~h}$ of life), or elevation of CRP serum levels (peak value $\geq 10 \mathrm{mg} / \mathrm{L}$ during the first $24 \mathrm{~h}$ of life).

When newborns were grouped according to the administration of fenoterol to the mother, no difference of monocyte L-selectin expression was found, whereas granulocyte L-selectin expression was reduced after tocolysis (309 versus $349, p<0.05$ ). However, the significance of this association was lost when acute bacterial infection was considered as a covariate.

Monocyte L-selectin expression was significantly increased after emergency cesarean delivery (median fluorescence: 346 versus $300, p<0.01$ ) compared with all other modes of delivery. There was no difference in L-selectin expression among infants born by elective cesarean, regular vaginal delivery, or assisted vaginal delivery $(p>0.1)$. Asphyxia (median fluorescence: 241 versus 307 ) and the presence of meconium-stained amniotic fluid (298 versus 308) were associated with a decreased monocyte L-selectin expression, although these differences attained significance $(p<0.05)$ only when acute infection was considered as a covariate. Granulocyte L-selectin expression was not affected by the mode of delivery, asphyxia, or meconium-stained amniotic fluid.

When umbilical cord blood samples $(n=127)$ were compared with blood samples from cubital veins of healthy adult volunteers $(n=28)$, L-selectin expression was significantly higher in granulocytes from adults (median fluorescence: 412 versus 343, $p<0.001$ ). This was also true when the nine neonates with acute bacterial infection were excluded or when the analysis was restricted to normal-term healthy newborns. Monocyte
$\mathrm{L}$-selectin expression in adults and newborns was indistinguishable (median fluorescence: 311 versus 303, $p$ > 0.1 ).

There was a considerable overlap in the range of relative L-selectin fluorescence intensities of both granulocytes and monocytes between the group of newborns with acute bacterial infection and the respective control group (Figs. 1 and 2). However, eight of nine newborns with acute bacterial infection had a granulocyte or monocyte L-selectin expression below the 10th percentile of the control group (sensitivity 89\%) compared with 19 of 118 controls (specificity 84\%). Calculations of negative and positive predictive values were not applicable due to the design of the study.

\section{DISCUSSION}

Although L-selectin down-regulation induced by a number of inflammatory signals in vitro has been established in murine and human neutrophils (10-17), this study now links reduced L-selectin expression of granulocytes and monocytes to activation of the immune system in vivo. L-Selectin down-regulation occurring during acute bacterial infection in vivo may be triggered either by bacterial products, such as FMLP-related peptides or lipopolysaccharides, or host-derived soluble mediators released during an ongoing acute systemic inflammatory response, such as cytokines, leukotriene B4, or C5a. However, the association of reduced L-selectin expression and acute bacterial infection may also be explained by the presence of a more immature phenotype in the circulation either as a risk factor or as a consequence of acute bacterial infection. The latter view is strengthened by the close mutual associations between acute bacterial infection, $\mathrm{I} / \mathrm{T}$ ratio, and $\mathrm{L}$-selectin expression.

Diminished L-selectin surface expression is supposedly linked with an impairment of L-selectin-dependent functions, such as extravasation, aggregation, or attachment to immobilized platelets $(1-7,14-16,19)$. However, because the loss of L-selectin surface expression observed in newborns with acute infection is partial rather than total, it may also be associated with focusing the extravasation of preactivated phagocytes on the most inflamed areas, where the reduced leukocyte L-selectin density is counteracted by an increased density of endothelial L-selectin ligands. This mechanism may balance the limited phagocyte supply from the bone marrow in newborns with acute bacterial infection, who typically present with leukopenia rather than leukocytosis (23).

Little is known about mechanisms involved in upregulating $\mathrm{L}$-selectin expression. To a variable extent, $\mathrm{L}$-selectin is expressed on all stages of granulocyte and monocyte precursor cells in the bone marrow (27), and L-selectin-negative granulocyte and monocyte precursors have been induced to express L-selectin by 3-d culture with IL-1, IL-2, IL-5, or IL-6 in vitro (28). How this may relate to complex events in vivo, e.g. emergency cesarean delivery, is presently unknown. Acute lack of 
oxygen itself does not appear to induce L-selectin upregulation, inasmuch as both a low umbilical cord artery $\mathrm{pH}$ and the presence of meconium in the amniotic fluid were found to be associated with a small L-selectin downregulation (monocytes) or with no change at all (granulocytes).

In a previous study of umbilical cord blood lymphocytes isolated by Percoll density-gradient centrifugation, we found lymphocyte L-selectin expression to be downregulated in acute bacterial infection of newborn infants in vivo (22). A number of leukocyte isolation procedures have been demonstrated to result in artificial loss of L-selectin from the surface of monocytes (29), whereas preparation methods involving sedimentation with dextran or hydroxyethyl starch have the additional risk of also inducing loss of neutrophil L-selectin $(13,29)$. Thus, analysis of unpurified blood is mandatory in correctly assessing L-selectin expression of all leukocyte populations simultaneously. Unfortunately, the lysing procedure used in this study did not allow accurate measurement of lymphocyte L-selectin expression due to variable contamination of the lymphocyte gate with residual unlysed red blood cells. This problem may be overcome in the future by increasing the time of lysis and changing the composition of the lysing solution or by doublefluorescence analysis using phycoerythrin-conjugated anti-CD45 MAb.

In fetal umbilical cord blood sampled percutaneously at 23 to 34 wk of gestation (21), eosinophils have been reported to constitute a large proportion of the granulocytes (median 46\%, range 10-88\%). However, seven of the eight samples had been from fetuses with isoimmune disease such as $\mathrm{Rh}$ incompatibility shown previously to be associated with increased eosinophil counts in fetuses of less than 34 wk gestation (30). Compared with those data published for specimens obtained antenatally (21), the values we found were significantly lower $(p<0.01)$ for both absolute eosinophil counts $(0-530 / \mu \mathrm{L}$, median $110 / \mu \mathrm{L})$ and eosinophil percentages of total granulocytes $(0-20 \%$, median $2 \%)$ in umbilical cord blood of newborns of less than 35 wk gestation $(n=27)$. Eosinophils from fetuses, term newborns, and adults have been reported to express L-selectin at about one third the level of neutrophils in the same specimen $(21,31)$. L-Selectin downregulation occurs also in eosinophils in response to calcium ionophores, but the role of physiologic stimuli has not been elucidated yet (30). In the postnatal specimens of the study presented here, eosinophils constituted only a small proportion of granulocytes irrespective of gestational age, and results are unlikely to have been confounded by varying proportions of eosinophils. Eosinophil percentages of Wright-Giemsa-stained blood smears were not related to granulocyte L-selectin surface expression measured by flow cytometry or to any parameter associated with altered granulocyte L-selectin expression. The question may be addressed prospectively using double-fluorescence measurements with anti-CD16 and anti-L-selectin MAb.
No single presumptive test has proved sufficient to identify the newborn infant with early-onset sepsis or to rule out bacterial infection in newborns presenting with ambiguous clinical signs. Most institutions use an array of different parameters to increase sensitivity and specificity of laboratory workup. Granulocyte and monocyte L-selectin expression both display an overlap of ranges of newborns with and without acute bacterial infection. However, when L-selectin expression of both granulocytes and monocytes was taken into account, the sensitivity and specificity obtained compared favorably with established laboratory parameters of early-onset sepsis (22-26). Determining the usefulness of L-selectin surface expression as a part of sepsis screens is beyond the scope of the study presented here, and the potential of this approach remains to be determined.

Acknowledgments. The authors thank Boris Metze and Reinhard Hartmann for expert assistance with statistics and data management and Günter Henze for use of the flow cytometry equipment.

\section{REFERENCES}

1. Abbassi O, Lane CL, Krater S, Kishimoto TK, Anderson DC, McIntire LV, Smith CW 1991 Canine neutrophil margination mediated by lectin adhesion molecule- 1 in vitro. J Immunol 147:2107-2115

2. Ley K, Gaethgens P, Fennie C, Singer MS, Lasky LA, Rosen SD 1991 Lectin-like cell adhesion molecule 1 mediates leukocyte rolling in mesenteric venules in vivo. Blood 77:2553-2555

3. Spertini O, Luscinskas FW, Gimbrone MA Jr, Tedder TF 1992 Monocyte attachment to activated human vascular endothelium in vitro is mediated by leukocyte adhesion molecule-1 (L-selectin) under nonstatic conditions. J Exp Med 175:1789-1792

4. von Andrian UH, Chambers JD, McEvoy LM, Bargatze REF, Arfors K-E Butcher EC 1991 Two-step model of leukocyte-endothelial cell interaction in inflammation: distinct roles for LECAM- 1 and the leukocyte $\beta 2$ integrins in vivo. Proc Natl Acad Sci USA 88:7538-7542

5. Watson SR, Fennie C, Lasky LA 1991 Neutrophil influx into an inflammatory site inhibited by a soluble homing receptor-IgG chimera. Nature 349:164-167

6. Simon SI, Rochon YP, Lynam EB, Smith CW, Anderson DC, Sklar LA 1993 $\beta 2$-Integrin and $\mathrm{L}$-selectin are obligatory receptors in neutrophil aggregation. Blood 82:1097-1106

7. Buttrum SM, Hatton R, Nash GB 1993 Selectin-mediated rolling of neutrophils on immobilized platelets. Blood 82:1165-1174

8. Hamann A, Jablonski-Westrich D, Jonas P, Thiele H-G 1991 Homing receptors reexamined: mouse LECAM-1 (MEL-14 antigen) is involved in lymphocyte migration into gut-associated lymphoid tissue. Eur J Immunol 21:2925 2929

9. Dawson J, Sedgwick AD, Edwards JCW, Lees P 1992 The monoclonal antibody MEL-14 can block lymphocyte migration into a site of chronic inflammation. Eur J Immunol 22:1647-1650

10. Spertini O, Kansas GS, Munro JM, Griffin JD, Tedder TF 1991 Regulation of leukocyte migration by activation of the leukocyte adhesion molecule- 1 (LAM-1) selectin. Nature 349:691-694

11. Berg M, James SP 1990 Human neutrophils release the Leu- 8 lymph node homing receptor during cell activation. Blood 76:2381-2388

12. Kishimoto TK, Jutila MA, Berg EL, Butcher EC 1989 Neutrophil Mac-1 and MEL-14 adhesion proteins inversely regulated by chemotactic factors. Science 245:1238-1241

13. Griffin JD, Spertini O, Ernst TJ, Belvin MP, Levine HB, Kanakura Y, Tedder TF 1990 Granulocyte-macrophage colony-stimulating factor and other cytokines regulate surface expression of the leukocyte adhesion molecule- 1 on human neutrophils, monocytes, and their precursors. J Immunol 145:576-584

14. Detmers PA, Powell DE, Walz A, Clark-Lewis C, Baggiolini M, Cohn ZA 1991 Differential effects of neutrophil-activating peptide 1/IL-8 and its homologues on leukocyte adhesion and phagocytosis. J Immunol 147:4211-4217

15. Kuijpers TW, Hakkert BC, Hart MH, Roos D 1992 Neutrophil migration across monolayers of cytokine-prestimulated endothelial cells: a role for platelet-activating factor and IL-8. J Cell Biol 117:565-572

16. Moser R, Olgiati L, Patarroyo M, Fehr J 1993 Chemotaxins inhibit neutrophil adherence to and transmigration across cytokine-activated endothelium: correlation to the expression of L-selectin. Eur J Immunol 23:1481-1487 
17. Jutila MA, Kishimoto TK, Butcher EC 1990 Regulation and lectin activity of the human neutrophil peripheral lymph node homing receptor. Blood 76:178 183

18. Jiang Y, Beller DI, Frendl G, Graves DT 1992 Monocyte chemoattractant protein-1 regulates adhesion molecule expression and cytokine production in human monocytes. J Immunol 148:2423-2428

19. Anderson DC, Abbassi O, Kishimoto TK, Koenig JM, McIntire LV, Smith CW 1991 Diminished lectin-, epidermal growth factor-, complement binding domain-cell adhesion molecule-1 on neonatal neutrophils underlies their impaired CD18-independent adhesion to endothelial cells in vitro. J Immuno 146:3372-3379

20. Török C, Lundahl J, Hed J, Lagercrantz H 1993 Diversity in regulation of adhesion molecules (Mac-1 and $\mathrm{x}$-selectin) in monocytes and neutrophils from neonates and adults. Arch Dis Child 68:561-565

21. Smith JB, Tabsh KMA 1993 Fetal neutrophils and eosinophils express normal levels of L-selectin. Pediatr Res 34:253-257

22. Bührer C, Luxenburger U, Metze B, Kattner E, Henze G, Dudenhausen JW, Obladen M 1993 Diminished cord blood lymphocyte L-selectin expression in neonatal bacterial infection. Eur J Pediatr 152:519-522

23. Manroe BL, Weinberg AG, Rosenfeld CR, Browne R 1979 The neonatal blood count in health and disease. I. Reference values for neutrophilic cells. J Pediatr 95:89-98
24. Mathers NJ, Pohlandt F 1987 Diagnostic audit of C-reactive protein in neonatal infection. Eur J Pediatr 146:147-151

25. Corrigan JJ 1974 Thrombocytopenia: a laboratory sign of septicemia in infants and children. J Pediatr 85:219-221

26. Tegtmeyer FK, Horn C, Richter A, van Wees J 1992 Elastase $\alpha_{1}$ proteinase inhibitor complex, granulocyte count, ratio of immature to total granulocyte count, and C-reactive protein in neonatal septicaemia. Eur J Pediatr 151:353 356

27. Kansas GS, Muirhead MJ, Dailey MO 1990 Expression of the CD11/CD18, leukocyte adhesion molecule 1 , and CD44 adhesion molecules during norma myeloid and erythroid differentiation in humans. Blood 76:2483-2492

28. Salmi M, Jalkanen S 1992 Regulation of L-selectin expression on cultured bone marrow leukocytes and their precursors. Eur J Immunol 22:835-843

29. Stibenz D, Bührer C 1994 Downregulation of L-selectin surface expression by various leukocyte isolation procedures. Scand J Immunol 39:59-63

30. Smith JB, Campbell DE, Ludomirsky A, Polin RA, Douglas SD, Garty BZ Harris MC 1990 Expression of the complement receptors CR1 and CR3 and the type II $\mathrm{Fc}_{\gamma}$ receptor on neutrophils from newborn infants and from fetuses with Rh disease. Pediatr Res 28:120-126

31. Smith JB, Kunjummen RD, Kishimoto TK, Anderson DC 1992 Expression and regulation of $\mathrm{L}$-selectin on eosinophils from human adults and neonates. Pediatr Res 32:465-471

\section{Announcement}

\section{Call for Abstracts}

The American Pediatric Society and The Society for Pediatric Research announce the abstract deadline for the 1995 Annual Meeting (May 8-11, 1995, San Diego Convention Center) has been set as January 3, 1995.

For further information, contact: APS/SPR Association Headquarters, 141 Northwest Point Blvd., P.O. Box 675, Elk Grove Village, IL 60009-0675, (708) 427-0205, Fax: (708) 427-1305. 